\title{
CONJUGATE POINT PROPERTIES FOR AN EVEN ORDER LINEAR DIFFERENTIAL EQUATION
}

\author{
GEORGE W. JOHNSON
}

\begin{abstract}
Consider an even order equation $D_{n} y=0$, where $D_{n}$ is defined recursively by $D_{0} y=y, a_{k, k+1} D_{k} y=\left\{\left(D_{k-1} y\right)^{\prime}-\sum_{i=1}^{k} a_{k i} D_{i-1}^{n} y\right\}$, where $a_{i j}$ is continuous on $[0, \infty), a_{i, i+1}>0, i=1, \cdots, n-1, a_{n, n+1} \equiv 1$, and $a_{i j} \equiv 0$ for $j>i+1$. The $k$ th conjugate point function $\eta_{k}(a)$ is defined and is shown to be a strictly increasing continuous function with domain $[0, b)$ or $[0, \infty)$. Extremal solutions are defined as nontrivial solutions with exactly $n-1+k$ zeros on $\left[a, \eta_{k}(a)\right]$, and are shown to exist, to have odd order zeros at $a$ and $\eta_{k}(a)$ and exactly $k-1$ odd order zeros in $\left(a, \eta_{k}(a)\right)$, thus establishing that $\eta_{k}(a)<\eta_{k+1}(a)$ if $\eta_{k}(a)<\infty$.
\end{abstract}

1. Introduction. The differential equation considered in this paper is defined recursively as follows. Let $a_{i j}$ be continuous functions on $[0, \infty)$ for $i, j=1, \cdots, n$ and let $a_{n, n+1} \equiv 1$. Further, let $a_{i, i+1}>0$ and let $a_{i j}=0$ if $j>i+1$. Let $A_{1}$ denote the set of all continuously differentiable functions defined in $[0, \infty)$. If $y$ is in $A_{1}$, let $D_{0} y=y$ and define

$$
D_{1} y=\left(1 / a_{12}\right)\left\{\left(D_{0} y\right)^{\prime}-a_{11} D_{0} y\right\} \text {. }
$$

If $A_{k}$ denotes the set of all functions $y$ for which $D_{k-1} y$ is continuously differentiable in $[0, \infty)$, then for $y$ in $A_{k}$, define $D_{k} y$ by

$$
D_{k} y=\frac{1}{a_{k, k+1}}\left\{\left(D_{k-1} y\right)^{\prime}-\sum_{i=1}^{k} a_{k i} D_{i-1} y\right\} \text {. }
$$

The differential equation under consideration is, for $n$ an even integer,

$$
D_{n} y=0 \text {. }
$$

This operator was studied extensively for $n=4$ by Barrett [1] and

Received by the editors June 29, 1973 and, in revised form, October 26, 1973. AMS (MOS) subject classifications (1970). Primary 34C10.

Key words and phrases. Extremal solution, conjugate point, zero of multiplicity $k$. 
Peterson [5], and for $n \geqq 4$ by Hinton [2].

In this paper we will restrict our attention to operators defined as follows. For the homogeneous boundary value problem

$$
D_{i-1} y\left(x_{j}\right)=0, \quad i=1, \cdots, m_{j}, j=1, \cdots, r,
$$

we will assume that there is no nontrivial solution to (1.3) and (1.4) with $m_{j}$ even, $1 \leqq j \leqq r$. We will refer to this assumption as Hypothesis (1.4).

If $D_{i-1} y(a)=0$ for $i=1, \cdots, k$ and $D_{k} y(a) \neq 0$ we say that $y$ has a zero of multiplicity $k$ at $x=a$. For convenience the term solution will be used to mean a nontrivial solution.

The purpose of this paper is to show that in general, the boundary value problem (1.4) determines the conjugate point properties of the operator $D_{n}$ in the following sense. Define the number

$$
\eta_{k}(a)=\inf N(k, a)
$$

where $N(k, a)$ denotes the set of all numbers $x>a$ for which there is a nontrivial solution of (1.3) having a zero at $a$, a zero at $x$ and at least $n-1+$ $k$ zeros in $[a, x]$. We can now state the main results.

Theorem 1. If $\eta_{k}(a)<\infty$, then $\eta_{k}(a)=\min N(k, a) ; \eta_{i}(a)<\infty$ for all $i \leqq k$, and $\eta_{i}(a) \leqq \eta_{i+1}(a)$.

If $y$ is a solution of (1.3) with a zero at $a$ and a zero at $\eta_{k}(a)$, then $y$ is said to be extremal for $\eta_{k}(a)$ if $y$ has at least $n-1+k$ zeros in $\left[a, \eta_{k}(a)\right]$. Theorem 1 guarantees the existence of such solutions for (1.3) if $\eta_{k}(a)<\infty$.

Theorem 2. If Hypothesis (1.4) holds, then an extremal solution, $y$, for $\eta_{k}(a)$ has exactly $n-1+k$ zeros in $\left[a, \eta_{k}(a)\right]$ with odd order zeros at $a$ and $\eta_{k}(a)$ and exactly $k-1$ odd order zeros in $\left(a, \eta_{k}(a)\right)$. Moreover $\eta_{k}(a)$ $<\eta_{k+1}(a)$.

Theorem 3. If Hypothesis (1.4) holds, then $\eta_{k}(x)$ is a strictly increasing continuous function of $x$ with domain $[0, b)$ or $[0, \infty)$.

2. General properties. The proof of Theorem 1 requires several lemmas.

Lemma 2.1. If $x$ is in $(0, \infty)$ then there is a $\delta>0$ such that no nontrivial solution of (1.3) has more than $n-1$ zeros in $(x-\delta, x+\delta)$.

The proofs of this lemma and the following lemma may be found in the 
paper of Hinton [2]. If $y_{1}, \cdots, y_{k}$ are solutions of (1.3), we define the wronskian $W_{k}(x)$ of $y_{1}, \cdots, y_{k}$ by $W_{k}(x)=\operatorname{det}\left(D_{i-1} y_{j}\right), i, j=1, \cdots, k$.

Lemma 2.2. If no nontrivial solution of (1.3) has more than $n-1$ zeros in $(a, b)$ then there exists a set of solutions $y_{1}, \cdots, y_{n}$ of (1.3) such that $W_{k}(x) \neq 0$ for $x$ in $(a, b), k=1, \cdots, n$.

Using Lemma 2.2 it is easy to establish the following basic property.

Lemma 2.3. If $\left\{y_{k}\right\}$ is a sequence of solutions of (1.3) for which $D_{i-1} y_{k}$ converges uniformly to $D_{i-1} y$ as $k \rightarrow \infty$, and if $y_{k}$ has a zero of multiplicity $m_{j}$ at $x_{k j}, j=1, \cdots, r$, and if $x_{k j} \rightarrow x$ as $k \rightarrow \infty$ for each $j=$ $1, \cdots, r$, then $y$ has a zero of multiplicity at least $m_{1}+\cdots+m_{r}$ at $x$.

We may now obtain the proof of Theorem 1 . The only significant case is the one in which there exists an infinite sequence $\left\{x_{j}\right\}$ converging to $\eta_{k}(a)$ and a sequence of solutions $y_{j}$ having $n-1+k$ zeros in $\left[a, x_{j}\right]$. We may assume with no loss of generality that each $y_{j}$ has zeros of multiplicity $m_{i}$ at $x_{i j}, i=1, \cdots, r$, with $x_{i j}=a, x_{r j}=x_{j}$. Moreover, if $z_{1}, \cdots, z_{n}$ are linearly independent solutions of (1.3), we may assume that $y_{j}=c_{1 j} z_{1}+$ $\cdots+c_{n j} z_{n}$ with $c_{1 j}^{2}+\cdots+c_{n j}^{2}=1$. This yields a subsequence $\left\{y_{j k}\right\}$ of $\left\{y_{j}\right\}$ for which $D_{i-1} y_{j k}$ converges uniformly to $D_{i-1} y$, where $y$ is a nontrivial solution of (1.3). Moreover, Lemma 2.3 and a elementary count establish that $y$ has at least $n-1+k$ zeros in $\left[a, \eta_{k}(a)\right]$ with a zero at $a$ and at $\eta_{k}(a)$. The rest of Theorem 1 follows easily.

3. Properties of $\eta_{k}(a)$. If $y$ is a solution of (1.3) with $n-1+k$ zeros at the points $x_{1}<x_{2}<\cdots<x_{r}$ of multiplicities $m_{i}$ at $x_{i}$, we define the number

$$
M(y)=\sum_{i \in I} m_{i}+\sum_{i \in J}\left[m_{i}-1\right],
$$

where $I$ is the set of indices, $i$, for which $m_{i}$ is even, and $J$ is the set of indices for which $m_{i}$ is odd. If $M(y)>n-2$, then as $M(y)$ is even, we must have $M(y) \geqq n$, which would contradict Hypothes is (1.4). Thus $M(y) \leqq n-2$ if $y \not \equiv$.

Lemma 3.1. If $y$ is extremal for $\eta_{k}(a)$, then $M(y)=n-2$.

Suppose to the contrary that $y$ is a solution with $n-1+k$ zeros at $x_{1}<\cdots<x_{r}$ and that $M(y) \leqq n-4$. We will establish that there exists a solution with at least $n-1+k$ zeros in $\left[x_{1}, x_{r}\right)$. Define a solution $y(x, \epsilon)$ 
of (1.3) by the conditions

$$
D_{i-1} y\left(x_{j}, \epsilon\right)=0, \quad 2 \leq j \leq r-1,
$$

for $i=1, \cdots, m_{j}$ if $m_{j}$ is even, and for $i=1, \cdots, m_{j}-1$ if $m_{j}$ is odd;

$$
D_{i-1} y\left(x_{r}-\epsilon, \epsilon\right)=D_{i-1} y\left(x_{r}\right)
$$

for $i=1, \cdots, m_{r}$ is even, and $i=1, \cdots, m_{r}+1$, if $m_{r}$ is odd;

$$
D_{i-1} y\left(x_{1}, \epsilon\right)=D_{i-1} y\left(x_{1}\right), \quad i=1, \cdots, q,
$$

where $q=n-\left[M(y)-m_{1}\right]$ if $m_{1}$ and $m_{r}$ are even, $q=n-\left[M(y)-m_{1}+2\right]$ if $m_{1}$ is even and $m_{r}$ is odd, $q=n-\left[M(y)-m_{1}+1\right]$ if $m_{1}$ is odd and $m_{r}$ is even, and $q=n-\left[M(y)-m_{1}+3\right]$ if $m_{1}$ and $m_{r}$ are both odd. In any case, $y$ is a solution of a boundary value problem of the form $D_{i-1} y\left(x_{j}\right)=$ $a_{i j}, i=1, \cdots, p_{j}, j=1, \cdots, r$, where $p_{i}$ is even, $p_{1}+\cdots+p_{r}=n$ and $a_{i j} \neq 0$ for at least one pair $i, j$. Using a standard compactness argument, we obtain that as $\epsilon$ tends to $0, D_{i-1} y(x, \epsilon)$ converges uniformly to $D_{i-1} y$. Then for $\epsilon$ sufficiently small, $y(x, \epsilon)$ must change sign near each odd order zero of $y$ (cf. Nicholson [4]). For each such $\epsilon, y(x, \epsilon)$ has $n-1+k$ zeros in $\left[x_{1}, x_{r}-\epsilon\right]$, and this completes the proof.

We may now observe that if $p$ is the number of odd order zeros of an extremal solution in $\left[a, \eta_{k}(a)\right]$ then $M(y)+p \geqq n-1+k$, so that $p \geqq k+1$. If $p>k+1$, and $m=1$, where $m$ denotes the multiplicity of the zero of $y$ at $\eta_{k}(a)$, then $y$ has $M(y)+p-1$ zeros in $\left[a, \eta_{k}(a)\right]$. But $M(y)+p-1 \geqq$ $(n-2)+(k+1)=n-1+k$, and this contradicts the fact that $y$ is extremal. If either $a$ or $\eta_{k}(a)$ is an even order zero of $y$, then $y(x, \epsilon)$ defined by (3.2), (3.3), (3.4) with $x_{r}=\eta_{k}(a), x_{1}=a$, will, for $\epsilon$ sufficiently small, have $n-1+k$ zeros in $\left[a, \eta_{k}(a)\right)$, and this holds for $p \geqq k+1$. Thus if $p>k+1$, then since $m$ is odd, we may regard $y$ as having $m-1$ zeros at $\eta_{k}(a)$ and obtain a contradiction.

4. Continuity of $\eta_{k}(a)$. Let $y$ be an extremal solution for $\eta_{k}(a)$ with zeros at $a=x_{1}<\cdots<x_{r}=\eta_{k}(a)$. For $2 \leqq i \leqq r-1$, let $y(x, \epsilon)$ satisfy the conditions (3.2). Define $D_{i-1} y\left(x_{1}+\epsilon, \epsilon\right)=D_{i-1} y\left(x_{1}\right), i=1, \cdots, m_{1}$. Observe that we have specified only $n-1$ homogeneous conditions on $y(x, \epsilon)$, so $y(x, \epsilon)$ is nontrivial. Then as $|\epsilon|$ tends to zero, $D_{i-1} y(x, \epsilon)$ converges uniformly to $D_{i-1} y^{*}(x)$ for $i=1, \cdots, n$, where $y^{*}$ is a nontrivial solution of (1.3). If $y^{*}$ and $y$ are linearly independent, then there is a linear combination of $y^{*}$ and $y$ satisfying the boundary conditions (1.4), which results 
in a contradiction. Thus $y^{*}$ is a constant multiple of $y$. Then for $|\epsilon|$ sufficiently small, $y(x, \epsilon)$ has $n-1+k$ zeros and $\eta_{k}(a+\epsilon)$ exists. Thus $\eta_{k}$ has an open domain. The same argument establishes that for any sequence $x_{i} \rightarrow a^{-}, \limsup \eta_{k}\left(x_{i}\right) \leqq \eta_{k}(a)$. If there exists a sequence $x_{1} \rightarrow a^{-}$such that $\lim \eta_{k}\left(x_{i}\right)=b<\eta_{k}(a)$, then with no loss of generality, the sequence $\left\{y_{i}\right\}$ of extremal solutions may be written as $y_{i}=c_{i 1} z_{1}+\cdots+c_{i n} z_{n}$ where $z_{1}, \cdots, z_{n}$ are linearly independent solutions of (1.3) and $c_{i 1}^{2}+\cdots+c_{i n}^{2}$ $=1$. Then a subsequence of $\left\{y_{i}\right\}$ will converge uniformly to a solution of (1.3) with $n-1+k$ zeros in $[a, b]$, which contradicts the definition of $\eta_{k}(a)$. Thus $\lim \eta_{k}(x)=\eta_{k}(a)$ as $x \rightarrow a^{-}$. A similar argument establishes the continuity of $\eta_{k}$ from the right. All that remains for the proof of Theorem 3 is to establish that $\eta_{k}$ is increasing.

Lemma 4.1. If $\eta_{k}(x)$ exists on $[a, b]$ and if $\eta_{k}(c)=\min \eta_{k}(x), x \in$ $[a, b]$, then $c=a$ or $c=b$.

For the proof, suppose to the contrary that $c$ is in $(a, b)$. Let $y$ be extremal for $\eta_{k}(c)$ with zeros $c=x_{1}<\cdots<x_{r}=\eta_{k}(c)$. Let $y(x, \epsilon)$ satisfy the conditions (3.2) for $j=1, \cdots, r-1$, and let $D_{i-1} y\left(x_{r}-\epsilon, \epsilon\right)=D_{i-1} y\left(x_{r}\right)$ for $i=1, \cdots, m_{r}$. Since only $n-1$ homogeneous conditions are specified, $y(x, \epsilon)$ is nontrivial. By uniform convergence, for $\epsilon$ sufficiently small there exists a point $d$ in $(c-\delta, c+\delta), c-\delta>a, c+\delta<b$, for which $y(x, \epsilon)$ has $n-$ $1+k$ zeros in $\left[d, \eta_{k}(c)-\epsilon\right]$, thus $\eta_{k}(d)<\eta_{k}(c)$ and $d$ is in $(a, b)$. This contradiction establishes the lemma.

Suppose now that $\eta_{k}$ exists on $[a, b]$, and suppose $\eta_{k}(b) \leqq \eta_{k}(a)$. Let $c=\sup S$, where $S$ denotes the set of all $x \geqq b$ for which $\eta_{k}(x) \geqq \eta_{k}(b)$. Clearly $S$ is nonempty and is bounded above by $\eta_{k}(b)$. It is not difficult to establish that $\eta_{k}(x)$ exists on $[a, c]$, and $\eta_{k}(c) \leqq \eta_{k}(b)$. Then for some $\epsilon>0, \eta_{k}(x)$ exists in $[c, c+\epsilon], c+\epsilon<\eta_{k}(b)$, and by definition of $c, \eta_{k}(x)$ $>\eta_{k}(b)$ for all $x$ in $(x, c+\epsilon)$. But this implies that there exists a point $d$ in $(a, c+\epsilon)$ such that $\eta_{k}(d)=\min \eta_{k}(x)$ for $x$ in $[a, c+\epsilon]$, contradicting Lemma 4.1. Thus it must be the case that if $\eta_{k}(x)$ exists for $x$ in $[a, b]$, then $\eta_{k}(a)<\eta_{k}(b)$. Another convergence argument establishes the fact that if $\eta_{k}(a)<\infty$, then $\eta_{k}(x)<\infty$ for all $x$ in $[0, a]$, and this completes the proof of Theorem 3 .

If we define the number $\eta_{k}^{-}(b)=\sup M(k, b)$, where $M(k, b)$ denotes the set of all $x<b$ for which there is a solution of (1.3) with a zero at $x$, a zero at $b$ and $n-1+k$ zeros in $[x, b]$, then we may obtain from Theorems 2 and 3 that $\eta_{k}^{-}$is the inverse function for $\eta_{k}$. 


\section{BIBLIOGRAPHY}

1. J. H. Barrett, Oscillation theory of ordinary linear differential equations, Advances in Math. 3 (1969), 415-509. MR $41 \# 2113$.

2. D. B. Hinton, Disconjugate properties of a system of differential equations, J. Differential Equations 2 (1966), 420-437. MR 34 \#856.

3. W. Leighton and Z. Nehari, On the oscillation of solutions of self-adjoint linear differential equations of the fourth order, Trans. Amer. Math. Soc. 89 (1958), 325-377. MR 21 \#1429.

4. L. S. Nicholson, Disconjugate systems of linear differential equations, J. Differential Equations 7 (1970), 570-583.

5. A. C. Peterson, Distribution of zeros of solutions of a fourth order differential equation, Pacific J. Math. 30 (1969), 751-764. MR 40 \#975.

DEPARTMENT OF MATHEMATICS, UNIVERSITY OF SOUTH CAROLINA, COLUMBIA, SOUTH CAROLINA 29208 